\title{
Remote monitoring in peritoneal dialysis: benefits on clinical outcomes and on quality of life
}

\author{
Sabrina Milan Manani ${ }^{1,2} @$ Michele Baretta ${ }^{1,2} \cdot$ Anna Giuliani $^{1,2} \cdot$ Grazia Maria Virzì $^{1,2} \cdot$ Francesca Martino $^{1,2}$. \\ Carlo Crepaldi ${ }^{1,2} \cdot$ Claudio Ronco $^{1,2,3}$
}

Received: 4 March 2020 / Accepted: 18 July 2020 / Published online: 10 August 2020

(c) Italian Society of Nephrology 2020

\begin{abstract}
Background Automated peritoneal dialysis (APD) has been proved benefit from remote monitoring (RM), but evidences are limited. In this study, we compared clinical outcomes and quality of life $(\mathrm{QoL})$ in two group of patients undergoing APD, with and without exposure of RM.

Methods This is a retrospective cohort study, comparing outcomes in two groups of APD patients monitored during 6 months with RM (group A: $n=35$ ) or standard care (group B: $n=38$ patients). In our clinical practice, we assign the RM system to patients who live more distant from the PD center or difficulty in moving. We evaluated emergency visits, hospitalizations, peritonitis, overhydration, and dropout. QoL was assessed with the Kidney Disease Quality of life-Short Form (KDQOL-SF). We included four additional questions focused on patient's perception of monitoring, safety and timely problems solution (Do you think that home-therapy monitoring could interfere with your privacy? Do you think that your dialysis sessions are monitored frequently enough? Do you think that dialysis-related issues are solved timely? Do you feel comfortable carrying out your home-based therapy?).

Results The case group presented a higher comorbidity score, according to Charlson Comorbidity Index (group A: 5.0; IQR 4.0-8.0 versus group B: 4.0; IQR 3.0-6.0) $(\mathrm{p}=0.042)$. The results in group A showed a reduction in the urgent visits due to acute overhydration (group A: $0.17 \pm 0.45$ versus group B: $0.66 \pm 1.36$ ) (p: 0.042 ) and in the number of disease-specific hospitalization (group A $n=2.0 ; 18.2 \%$ versus group $B \mathrm{n}=7.0 ; 77.8 \%)(\mathrm{p}=0.022)$. We did not find any difference between the two groups in terms of hospitalization because of all-cause, peritonitis, overhydration, and dropout. The analysis of KDQOLSF subscales was similar in the two groups; on the contrary, the answers of our pointed questions have showed a significant difference between the two groups (group A: 100 IQR 87.5-100.0 versus group B 87.5; IQR 75.0-100.0) (p: 0.018).

Conclusion RM improved clinical outcomes in PD patients, reducing the emergency visits and the hospitalizations, related to nephrological problems, especially in patients with higher comorbidity score. The acceptance and satisfaction of care were better in patients monitored with RM than with standard APD.
\end{abstract}

Keywords Remote monitoring $\cdot$ APD $\cdot$ Telemedicine $\cdot$ Clinical outcomes $\cdot$ Quality of life

Sabrina Milan Manani

sabrina.milan@aulss8.veneto.it

1 Department of Nephrology, Dialysis and Transplantation, St. Bortolo Hospital, Via Rodolfi, 37, 36100 Vicenza, Italy

2 IRRIV - International Renal Research Institute of Vicenza, Via Rodolfi, 37, 36100 Vicenza, Italy

3 Università degli Studi di Padova, Via 8 Febbraio 1848, 2, 35122 Padova, Italy

\section{Introduction}

The world population is getting older and is increasingly living with chronic diseases. Heart failure (HF), chronic pulmonary obstructive disease (COPD), hypertension, diabetes, and end stage renal disease (ESRD) are life-threatening chronic diseases and they are a global pandemic: over 26 million people are affected by HF and 2 million people by chronic kidney disease (CKD) worldwide [1].

These are long-term conditions, associated with high prevalence and high health costs. In fact, they are characterized by periodic exacerbations and recurrent 
hospitalizations. Despite advances of medicine, patients continue to experience poor survival and health-related quality of life (QoL) [2]. Moreover, most of these patients live in remote and rural areas, where medical support may not be available [3].

Novel healthcare strategies, such as multidisciplinary care and telemedicine, are required to support these conditions. In fact, care coordination is becoming an important issue in a population suffering from multiple pathologies [4-6].

Telemedicine, providing services at a distance, can tackle the challenges to healthcare systems, by shortening waiting lists, optimizing the use of resources, reducing hospital stays and improving the QoL of chronically ill patients $[7,8]$.

Telemedicine can be particularly helpful in patients affected by ESRD who are undergoing peritoneal dialysis (PD). This is a home-based therapy that allows for a greater independence from the hospital compared to the in-center hemodialysis (HD), as well as an improved QoL.

Telemonitoring or remote monitoring (RM), an application of telemedicine, can provide a closer follow-up of outpatients compared to usual care, with a daily monitoring of PD treatments and tailoring of PD prescriptions [9].

In particular, it has been proven that Automated Peritoneal Dialysis (APD) can benefit from RM, through earlier recognition and correction of problems, thereby reducing the frequency of in-person visits [10].

In a recent study, Viglino et al. describe their experience with Videodialysis, a new telemedicine system that allows to overcome physical, cognitive and psychological barriers to PD [7].

However, evidence is limited, and Wallace et al. [11] suggest that further studies should focus on direct comparison between telehealth and standard care regarding clinical outcomes and patients' QoL.

The present study was conducted in 73 stable PD patients treated with APD, with a without RM, regularly followed in our department during 6 months period, between March and August 2019. The aim of the study was to evaluate clinical outcomes, such as number of visits, hospitalizations, peritonitis, overhydration, dropout, and QoL between the two groups.

\section{Methods}

\section{APD program in Vicenza, Italy PD center}

In Vicenza PD center, APD is performed using two different software systems, equally allocated between all the patients. Recently, both the APD systems have been integrated with an internet connection (RM system) that enables you to remotely monitor the dialysis treatments and to modify the APD prescription with no need for bringing the card to the
PD center (Claria ${ }^{\mathrm{TM}}$, connected to Sharesource platform, Baxter Healthcare, Deerfield, Illinois and Sleep-safe Harmony connected to home bridge Connectivity PD, Fresenius Medical Care, Bad Homburg, Germany). The Sharesource platform reports also some clinical data registered by patients, such as blood pressure and weight.

At the beginning of the study, in our PD center some patients were treated with the traditional card-system and some patients with the new RM system.

Regarding the RM system, in our PD center the nurses check the platform every day. According to their observation, the physicians perform a daily check of treatments with significant issues and a weekly check of treatments without serious problems.

Regarding the traditional card system, dialysis sections were reviewed during the routinely planned hospital visits every 3 months, and in case of unplanned visits due to complications experienced by the patients, or identified by the care team in a phone conversation.

In our clinical practice, we assign the RM system to patients who live more distant from the PD center or difficulty in moving.

In both APD systems the dialysis prescription followed the international guidelines for maximizing PD clearances [12-15].

\section{Study design}

This is a retrospective case-control, single-center study, comparing clinical outcomes and QoL in two current groups of patients undergoing APD from 01.03.2019 to 30.08.2019 in the PD center at San Bortolo Hospital, in Vicenza, Italy.

We enrolled all the patients-over 18 years old and treated with APD for more than 3 months (prevalent patients) - in our PD center in the same 6-month period. All procedures were in accordance with the Helsinki Declaration. The protocol and consent form were approved by the Ethics Committee of San Bortolo Hospital. All patients were informed about the experimental protocol and the objectives of the study and they all gave written informed consent. None of the patients denied the written consent; no enrolled patient was excluded from the study.

We divided the patients in two groups: the case group (group A) included 35 patients monitored with RM (RM-APD) and the control group (group B) consisted of 38 patients treated with traditional APD without RM (traditional-APD).

Laboratory data and dialysis-related parameters were recorded at the end of the observation period. Total renal plus peritoneal clearance was utilized to calculate weekly $\mathrm{Kt} / \mathrm{V}_{\text {urea }}$ and weekly creatinine clearance as PD adequacy parameters [13]. 
Comorbidity was assessed using the Charlson Comorbidity Index, "age-adjusted" [16].

\section{Outcomes}

As outcomes, we evaluated in both groups the number of urgent visits, the number of all-cause and disease-specific hospitalizations, the number of all-cause and disease-specific hospital days, the cases of peritonitis, the level of overhydration, the number of dropouts. Furthermore, we considered the QoL for all the patients.

We considered urgent visits due to acute over hydration with edemas or dyspnea, exit site infections, peritonitis or other issues such as hypertension or catheter dysfunction.

Disease-specific hospitalizations were caused by fluid overload, peritonitis or exit-site infections, dialysis access dysfunction.

Peritonitis was defined according to the criteria of International Peritoneal Dialysis Society (ISPD), as a cloudy effluent with at least 100 leukocytes/ $\mu \mathrm{L}$, of which more than $50 \%$ were polymorph nuclear cells, generally with abdominal pain and/or a positive effluent culture [17].

Hydration was assessed by a bio-impedance spectroscopy device, the Body Composition Monitor (BCM) (Fresenius Medical Care).

The 10th and 90th percentile of Absolute $\Delta$ Tissue Hydration $(\mathrm{A} \Delta \mathrm{TH})$ in the healthy population $(-1.1$ and $+1.1 \mathrm{~L}$ respectively) representing thus limits of "normohydration". The patients with $\mathrm{A} \Delta \mathrm{TH}$ higher than $+1.1 \mathrm{~L}$ were considered over hydrated [18].

As dropout, we recorded the number of shifts from PD to hemodialysis (HD), deaths and transplantations.

We also evaluated the treatment compliance in the two groups in the 3 months of dialysis preceding the start of observation period and during the six-month period of the study. In particular, we registered the number of treatments not performed in every group.

QoL was assessed at baseline with the Kidney Disease Quality of life-Short Form (KDQOL-SF). This is a selfreport measure developed for patients with kidney disease and on dialysis. The instrument includes multi-item scales with precoded numeric values for responses. The scoring procedure transforms the numeric values of items to a 0-100 range, with higher transformed scores always reflecting better QoL. The Italian version was validated in an Italian population [19-21].

We also added to KDQOL-SF four questions that we have realized to focus on PD patients QoL, in particular to analyze perception of monitoring, safety and timely problem solution. A similar scoring algorithm was used to calculate scores ranging from 0 to 100 , with 100 representing the highest QOL. This internal questionnaire is reported in Table 1.

\section{Statistical analysis}

Statistical analysis was performed using the SPSS Software package. Categorical variables were expressed as percentages; continuous variables were expressed as mean \pm standard deviation (parametric variables) or median and interquartile range (IQR) (nonparametric variables). The Mann-Whitney U test o T test were used for comparison of two groups, as appropriate. Categorical variables were compared using $\chi^{2}$ test. Correlation coefficients were calculated with the Spearman's rank or Pearson's test, as appropriate. A $p$ value of $<0.05$ was considered statistically significant.

\section{Results}

The case group included 35 patients, treated by RM-APD and the control group included 38 patients that performed traditional APD.

Both groups were current cohorts and they were followed for 6 months from 01.03.2019 to 30.08.2019.

The Table 2 summarizes the baseline characteristics of the two groups. In particular the clinical data were similar in the two groups, but group A presented a higher co-morbidity score compared to group $\mathrm{B}$, according to the Charlson Comorbidity Index (5.0; IQR 4.0-8.0 versus 4.0; IQR 3.0-6.0) $(\mathrm{p}=0.042)$.

The results showed a lower number of urgent visits in group A compared to group B, although not statistically significant difference was found (mean $1.14 \pm 1.33$ versus mean $1.78 \pm 2.05)(\mathrm{p}=0.095)$.

Furthermore, in the case group there was a reduction in the number of disease-specific hospitalizations (group A $\mathrm{n}=2.0 ; 18.2 \%$ versus group $\mathrm{B} \mathrm{n}=7.0 ; 77.8 \%)(\mathrm{p}=0.022)$. However, we did not find any differences between the two
Table 1 Internal questionnaire to investigate patient's perception of monitoring, safety and timely problem solution

\begin{tabular}{l}
\hline \\
\hline Do you think that home-therapy monitoring could interfere with your privacy? \\
Do you think that your dialysis sessions are monitored frequently enough? \\
Do you think that dialysis-related issues are solved timely? \\
Do you feel comfortable carrying out your home-based therapy?
\end{tabular}


Table 2 Baseline characteristics of patients in group $A$ (RM-APD) and group B (without RM-APD)

\begin{tabular}{llll}
\hline Baseline characteristics & $\begin{array}{l}\text { Group A } \\
\text { Patients with RM-APD }\end{array}$ & $\begin{array}{l}\text { Group B } \\
\text { Patients without RM-APD }\end{array}$ & p-value \\
\hline Patients, number & 35 & 38 & \\
Age, years, median (IQR) & $62.8(44.7-77.1)$ & $57.9(50.0-73.1)$ & $0.930^{1}$ \\
Dialysis vintage, months, median (IQR) & $24.8(10.0-48.7)$ & $25.1(15.3-42.6)$ & $0.619^{1}$ \\
Gender, men, \% & 77 & 71 & $0.554^{2}$ \\
BMI, mean \pm SD & $27.24 \pm 5.00$ & $25.64 \pm 4.10$ & $0.189^{1}$ \\
Diuresis, mL, median (IQR) & $800(75-1350)$ & $1000(500-1258)$ & $0.440^{1}$ \\
Hemoglobin, g/L, mean \pm SD & $113.2 \pm 16.1$ & $115.5 \pm 12.5$ & $0.398^{1}$ \\
Albumin, g/dL, median (IQR) & $3.8(3.6-4.0)$ & $3.9(3.6-4.0)$ & $0.760^{1}$ \\
Total wKt/V, mean \pm SD & $1.76 \pm 0.60$ & $1.72 \pm 0.42$ & $0.928^{1}$ \\
Total wClCr, L/week, median (IQR) & $53.2(29.9-75.1)$ & $53.7(39.9-74.6)$ & $0.938^{1}$ \\
Charlson comorbidity index, median (IQR) & $5.0(4-8)$ & $4.0(3-6)$ & $0.042^{1}$ \\
Caregiver-assisted PD, number (\%) & $6(17.1)$ & $4(10.5)$ & $0.42^{2}$ \\
\hline
\end{tabular}

Values represent numbers, percentages $(\%)$, mean $\pm \mathrm{SD}(\mathrm{SD}$, standard deviation) or medians (IQR, interquartile range)

$R M$ remote monitoring, $A P D$ automated peritoneal dialysis, $B M I$ body mass index, total $w K t / V$ total (renal and peritoneal) weekly $\mathrm{Kt} / \mathrm{V}_{\text {urea }}$, total $w C l C r$ total (renal and peritoneal) weekly creatinine clearance

${ }^{1}$ Mann-Whitney U test

${ }^{2}$ Pearson's $\chi^{2}$ test groups regarding the number of all-cause hospitalizations (group A $\mathrm{n}=11.0 ; 31.4 \%$ versus group $\mathrm{B} n=9.0 ; 23.7 \%$ ) $(\mathrm{p}=0.632)$, days of all-cause hospitalization (group A 5.0; IQR 4.0-11.5 versus group B 10.0; IQR 7.0-20.0) $(\mathrm{p}=0.552)$, and days of disease-specific hospitalization (group A 7.0; IQR 5.5-8.5 versus group B 9.5; IQR 7.5-11.5) $(\mathrm{p}=0.093)$.

Similarly, there was no significant difference in terms of the level of overhydration expressed by BCM (group A 2.2; IQR 1.0-3.5 versus group B 2.1; IQR 0.8-3.3) ( $\mathrm{p}=0.720)$, the number of peritonitis (group $\mathrm{A} n=3.0 ; 8.5 \%$ versus group $B \mathrm{n}=2.0 ; 5.3 \%)(\mathrm{p}=0.579)$, and the number of dropouts (group A $n=4.0 ; 11.4 \%$ versus $n=4.0 ; 10.5 \%$ ) $(\mathrm{p}=0.904)$.

The different outcomes in the two groups are reported in the Fig. 1.

We evaluated the different issues that required urgent visits and we observed a statistically lower number of urgent visits due to acute overhydration $(\mathrm{p}=0.042)$ and exit site infections $(\mathrm{p}=0.045)$ in group A compared to group $\mathrm{B}$. The urgent visits caused by peritonitis or other causes were similar in the two groups $(\mathrm{p}=0.581$ and $\mathrm{p}=0.823$, respectively). The Table 3 summarizes the clinical problems that required urgent visits.

During the time of observation, we recorded eight dropouts, four in group A and four in group B. In particular, in the case group, three patients died and one had a kidney transplant, whereas in the control group, one patient was shifted from PD to HD due to the peritoneal function loss and three patients had a kidney transplant.
The treatment compliance was similar in group A and group B before and during the observational study (number of treatments not performed group $A=60$ versus group $B=92)(p=0.67)$.

In addition, we compared the results of the KDQOLSF test and the internal QOL PD questionnaire of the two groups. All KDQOL-SF subscales had similar scores in group A and group B. However, the answers to the internal QoL PD questionnaire showed a significant difference between the two groups $(p=0.018)$. In particular, group A felt that dialysis treatments were monitored more frequently, and there was a quicker solution of dialysis problems compared to group B. Moreover, the patients of the case group felt safer regarding their home dialysis compared to the patients of the control group and the dialysis monitoring did not interfere with privacy in group A more than in group B (Table 4).

\section{Discussion}

The present study investigated the benefits of telemedicine on clinical outcomes and on QoL in patients receiving PD. This new tool has been recently developed to allow the follow up of an increasing population affected by different chronic diseases, such as diabetes, HF, CKD. The most important aims of telemedicine are to improve and tailor healthcare as well as reduce costs. In fact, there is increasing evidence that supports the use of telemonitoring of outpatients. 


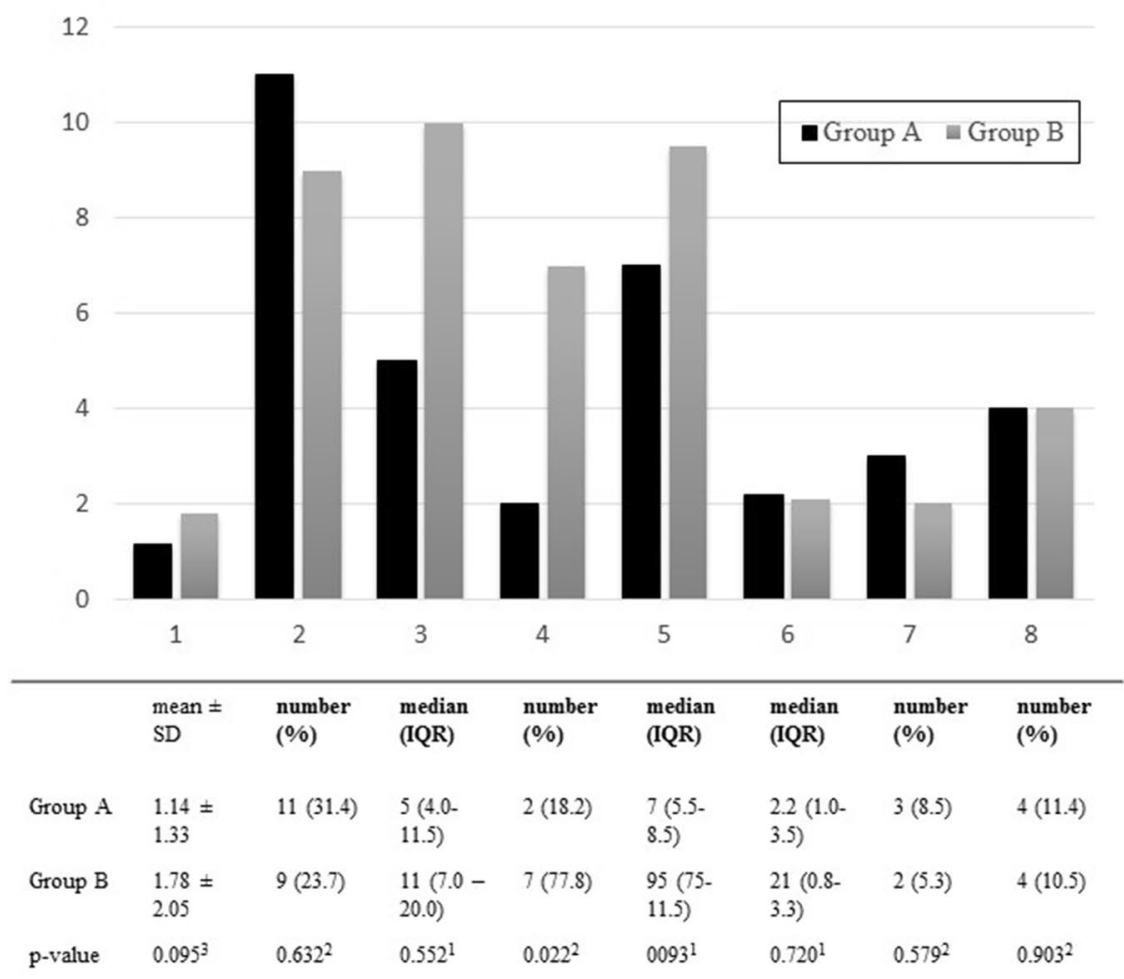

1, Urgent Visits; 2, Hospitalizatiomns; 3, Days of Hospitalization; 4, Desease-Specific Hospitalizations; 5, Days of Deasese-Specific Hospitalization; 6, Body Composition Monitor (BCM); 7, Peritonitis; 8, Drop-out; RM, Remote Monitoring; APD, Automated Peritoneal Dialysis; Values represent numbers, percentages $(\%)$, means $=\mathrm{SD}\left(\mathrm{SD}\right.$, standard deviation) or medians (IQR, interquartile range); ${ }^{1} \mathrm{Mann}$-Whitney U test. ${ }^{2} \mathrm{Pearson}$ 's $\chi^{2}$ test. ${ }^{3} \mathrm{~T}$ test .

Fig. 1 Clinical outcomes in group A (RM-APD) and group B (without RM-APD). $R M$ remote monitoring, $A P D$ automated peritoneal dialysis, $B C M$ body composition monitor, $n$ number, $\%$ percentage,
$S D$ standard deviation, IQR interquartile range; ${ }^{1}$ Mann-Whitney $\mathrm{U}$ test; ${ }^{2}$ Pearson's $\chi^{2}$ test; ${ }^{3} \mathrm{~T}$ test
Table 3 Issues that required urgent visits in group A (with RM-APD) and in group B (without RM-APD)

\begin{tabular}{llll}
\hline Issues & $\begin{array}{l}\text { Group A } \\
\text { Patients with RM-APD }\end{array}$ & $\begin{array}{l}\text { Group B } \\
\text { Patients without RM- } \\
\text { APD }\end{array}$ & p-value \\
\hline Overhydration, mean \pm SD & $0.17 \pm 0.45$ & $0.66 \pm 1.36$ & $0.042^{1}$ \\
Exit site infections, mean \pm SD & $0.17 \pm 0.56$ & $0.42 \pm 0.85$ & $0.045^{1}$ \\
Peritonitis, mean \pm SD & $0.08 \pm 0.40$ & $0.05 \pm 0.44$ & $0.581^{1}$ \\
Other issues, mean \pm SD & $0.65 \pm 0.48$ & $0.63 \pm 0.48$ & $0.823^{1}$ \\
\hline
\end{tabular}

$R M$ remote monitoring, $A P D$ automated peritoneal dialysis, \% percentage

${ }^{1} \mathrm{~T}$ test

For example, in diabetes care the new tools that allow remote and more frequent medication adjustments improve glycated hemoglobin [22].

Similarly, a cardio-device has been shown to be effective in reducing the number of hospitalizations for $\mathrm{HF}$ [23].

However, Eurlings et al. [24] in their review underline that, even though new technology is evolving, the evidence is conflicting, mainly due to a lack of uniform methods to evaluate telehealth and standard care.
In our PD center we adopted RM from 2016 and we studied various aspects of this innovative method.

Firstly, we compared APD incident and prevalent patients undergoing RM-APD with APD incident and prevalent patients treated with traditional APD with the same software system. The length of observation for every patient was 6 months. We observed that the APD prescriptions were modified more frequently in RM-APD versus traditional APD in incident and prevalent patients. Visits were significant less in RM-APD than in traditional APD 
Table 4 Kidney disease quality of life-short form (KDQOL-SF) and quality of life (QoL) PD questions in group A (with RM-APD) and in group B (without RM-APD)

\begin{tabular}{lccc}
\hline Scale (item) & $\begin{array}{l}\text { Group A } \\
\text { Patients with RM-APD }\end{array}$ & $\begin{array}{l}\text { Group B } \\
\text { Patients without RM-APD }\end{array}$ & p-value \\
\hline Burden of kidney disease & $43.8(25.0-68.8)$ & $50.0(31.3-68.8)$ & $0.332^{3}$ \\
Quality of social interaction & $73.3(63.3-85.8)$ & $86.7(68.3-91.7)$ & $0.294^{3}$ \\
Cognitive function & $80.0(66.7-95.0)$ & $83.3(70.0-98.3)$ & $0.914^{3}$ \\
Symptoms & $77.1(60.8-85.4)$ & $83.3(72.9-91.7)$ & $0.288^{3}$ \\
Effects of kidney disease & $67.2(53.9-84.4)$ & $73.4(55.5-91.4)$ & $0.707^{3}$ \\
Sexual function & $100.0(100.0-100.0)$ & $100.0(96.9-100.0)$ & $0.197^{3}$ \\
Sleep & $65.0(50.0-75.5)$ & $75.0(55.0-85.0)$ & $0.591^{3}$ \\
Social support & $83.3(66.7-100.0)$ & $83.3(66.7-100.0)$ & $0.715^{3}$ \\
Work status & $50.0(37.5-100.0)$ & $50.0(0.0-100.0)$ & $0.689^{3}$ \\
Patient satisfaction & $83.3(66.7-100.0)$ & $83.3(66.7-100.0)$ & $0.999^{3}$ \\
Dialysis staff encouragement & $100.0(100.0-100.0)$ & $100.0(87.5-100.0)$ & $0.167^{3}$ \\
QoL peritoneal questions & $100(87.5-100.0)$ & $87.5(75.0-100.0)$ & $0.018^{3}$ \\
\hline Values represent medians (IQR, interquartile range) & & \\
$P D$ peritoneal dialysis, $R M$ remote monitoring, APD automated peritoneal dialysis & \\
${ }^{3}$ T test &
\end{tabular}

for incident patients, while no significant difference was observed between prevalent populations [22].

Subsequently, we investigated the benefits of the prolonged use of RM-APD in 2-year experience.

We observed 42 RM-APD patients during a median length of 13.28 months per patient. The control group consisted of 42 patients, previously treated by traditional APD with the same software system, for APD with card system. Our results showed that changes in APD prescription were almost double in the case group (RM) compared to the control group. The need for in-person visits and nocturnal alarms were significantly less in RM-APD than in traditional APD. The distance travelled by patients in case of RM-APD was reduced by $1134 \mathrm{~km}$ with a time saving of $1554 \mathrm{~min}$ for patients. The overall cost reduction for the PD center in terms of time/nurse and time/physician was 2647 and $3673 \mathrm{~min}$, respectively. Furthermore, all patients found the TM system easy to use and they were satisfied with the high level of interaction with the careteam and with the possibility of timely solving technical problems [23].

In the present study, we would like to demonstrate that RM of APD allows a knowledge-based management, reducing the urgent events and improving clinical outcomes.

In two previous studies, we investigated the benefits of RM-APD using an historical cohort of patients treated with traditional APD system as comparison group. However, comparing current versus historical cohort there could be a bias of analysis related to different time of observation and different clinical practice $[25,26]$.

In this study, we analyzed data of patients treated with traditional APD and RM-APD during the same period of observation, avoiding potential differences between groups separated in time, in particular because of differences in concomitant standards of care [27].

Moreover, we evaluated the improvement of clinical outcomes obtained by RM, such as the reduction of urgent visits in the PD center, hospitalization, overhydration, peritonitis, and dropouts in two current groups of patients. Finally, we analyzed the patients' QoL and satisfaction with their own modality of treatment and monitoring.

The biochemical and PD adequacy parameters were similar in the two groups and these data demonstrate the same application of international criteria for the dialysis prescription. On the contrary, the Charlson Comorbidity Index was worse in the case group than in the control group.

In fact, in our clinical practice, we give the RM system first to the patients with major comorbidities, to provide a closer and prompter care. Previous studies have demonstrated that sociodemographic and clinical characteristics do not negatively influence ability to master the technology and that elderly patients adapt quickly to telehealth [28, 29].

In this study, the new tool helped reduce the urgent visits in the group with more comorbidities and in particular, there was a reduction of acute overhydration episodes and exit site infections.

The reduction of exit-site infections could be easier related with forms of virtual caregiver-based telemedicine such as Videodialysis or with careful PD training, performed by specialized nurses [7,30]. Future larger and prospective studies will be useful to explain better the effect of RM-APD on exit site infections.

Indeed, the reduction of acute overhydration episodes suggests that an early identification and a timely trouble shooting of PD-related issues avoid the development of relevant clinical problems [31]. 
The same conclusion could explain the second important result of this study, the reduction of disease-specific hospitalizations in patients receiving RM-APD.

Gallar et al. obtained a similar result following the patients with a monthly teleconsultation and recently, Sanabria et al. observed a lower hospitalization rate and fewer hospital days in a Baxter Renal care Service unit in Colombia $[32,33]$.

At last, the reduction of urgent events could allow better planning of the care activity.

Regarding the similar number of treatments not performed in the two groups, we conjecture that a positive relationship between patients and team-care could optimize the patient's compliance. However, in our experience RM system makes easier to give a timely and close support to our patients as well as to monitor their compliance.

Moreover, we investigated the potential benefits of RM on QoL.

According to the KDQL-SF scale, previous studies demonstrated that RM groups believed that they received more staff encouragement and that their pain was improved. The other aspects of QoL did not change with or without RM [34].

Group A and B of our study presented similar scores in all KDQOL-SF subscales. Indeed, the KDQL-SF is an instrument developed for individuals with kidney disease and on dialysis, but there are not specific questions assessing the utility of home dialysis monitoring. For this reason, we designed an internal questionnaire, to better evaluate the usability of the telemonitoring system, similarly to Martinez Garcia et al. [35] in their research article.

Based on these specific questions, the patients treated with RM-APD indicated a good acceptance and satisfaction when monitored closely and frequently.

In conclusion, our data demonstrated that RM technology improved clinical outcomes in PD patients, reducing the emergency visits and hospitalizations related to nephrological problems, even for patients with a worse comorbidity score.

The patient's acceptance and satisfaction of care were better, when monitored with RM than with standard APD.

Nevertheless, we acknowledge the limitations of the small sample size of this mono-center and retrospective study. For the first time, we describe clinical outcomes of an Italian population in APD and introduce some considerations on patient's QoL. In this context, the present study could provide the basis for larger studies that may clarify the utility and the acceptance of RM-APD for PD patients, as well as for their caregivers and the care-team.

Author contributions SMM: conception of the study, interpretation of data and drafting the article. MB: management of patients. AG: conception of the study, interpretation of data and drafting the article. GMV: interpretation of data and statistical analysis. FM: interpretation of data and statistical analysis. CC: final approval of the draft and management of patients. CR: providing intellectual content of critical importance to this work. All authors approved the final version of this paper.

Funding The authors did not use funding sources.

\section{Compliance with ethical standards}

Ethical approval All procedures were in accordance with the Helsinki Declaration. The protocol and consent form were approved by the Ethics Committee of San Bortolo Hospital. All patients were informed about the experimental protocol and the objectives of the study and they all gave written informed consent. None of the patients denied the written consent.

Conflict of interest C. Ronco is a consultant for Alere, and a member of speakers bureau for Abbot Diagnostics. The other authors declare no conflict of interest.

\section{References}

1. Rojahn K, Laplante S, Sloand J, Main C, Ibrahim A, Wild J, Sturt N, Areteou T, Johnson KI (2016) Remote monitoring of chronic diseases: a landscape assessment of policies in four European countries. PLoS One 11:e0155738

2. Javaid MM, Khan BA, Subramanian S (2019) Peritoneal dialysis as initial dialysis modality: a viable option for late-presenting end-stage renal disease. J Nephrol 32:51-56

3. Ding H, Jayasena R, Maiorana A, Dowling A, Chen SH, Karunanithi M, Layland J, Edwards I (2017) Innovative Telemonitoring Enhanced Care Programme for Chronic Heart Failure (ITEC-CHF) to improve guideline compliance and collaborative care: protocol of a multicentre randomised controlled trial. BMJ Open 7:e017550

4. Thilly N, Chanliau J, Frimat L, Combe C, Merville P, Chauveau P, Bataille P, Azar R, Laplaud D, Noel C, Kessler M (2017) Cost-effectiveness of home telemonitoring in chronic kidney disease patients at different stages by a pragmatic randomized controlled trial (eNephro): rationale and study design. BMC Nephrol 18:126

5. Noppakun K, Kasemset T, Wongsawad U, Ruengorn C, Thavorn K, Sood MM, Nochaiwong S (2020) Changes in serum albumin concentrations during transition to dialysis and subsequent risk of peritonitis after peritoneal dialysis initiation: a retrospective cohort study. J Nephrol. https://doi.org/10.1007/s40620-02000716-1

6. Garofalo C, Borrelli S, De Stefano T, Provenzano M, Andreucci M, Cabiddu G, La Milia V, Vizzardi V, Sandrini M, Cancarini G, Cupisti A, Bellizzi V, Russo R, Chiodini P, Minutolo R, Conte G, De Nicola L (2019) Incremental dialysis in ESRD: systematic review and meta-analysis. J Nephrol 32:823-836

7. Communication from the Commission to the European Parliament, The Council, The European Economic and Social Committee and the Committee of the Regions on telemedicine for the benefit of patients, healthcare systems and society. Brussels, 4.11.2008. $\operatorname{COM(2008)689~final.~http://www.senato.it/web/docuo~}$ rc2004.nsf/4d9255edaa0d94f8c12576ab0041cf0a/6b7767d529 b59effc1257501004b8a82/\$FILE/COM2008_0689_IT.pdf 
8. Basile C, Casino FG, Aucella F (2019) Incremental hemodialysis, a valuable option for the frail elderly patient. J Nephrol 32:741-750

9. Rosner MH, Ronco C (2012) Remote monitoring for continuous peritoneal dialysis. Contrib Nephrol 178:68-73

10. Drepper VJ, Martin PY, Chopard CS, Sloand JA (2018) Remote patient management in automated peritoneal dialysis: a promising new tool. Perit Dial Int J Int Soc Perit Dial 38:76-78

11. Wallace EL, Rosner MH, Alscher MD, Schmitt CP, Jain A, Tentori F, Firanek C, Rheuban KS, Florez-Arango J, Jha V, Foo M, de Blok K, Marshall MR, Sanabria M, Kudelka T, Sloand JA (2017) Remote patient management for home dialysis patients. Kidney Int Rep 2:1009-1017

12. Blake P, Burkart JM, Churchill DN, Daugirdas J, Depner T, Hamburger RJ, Hull AR, Korbet SM, Moran J, Nolph KD (1996) Recommended clinical practices for maximizing peritoneal dialysis clearances. Perit Dial Int J Int Soc Perit Dial 16:448-456

13. Dombros N, Dratwa M, Feriani M, Gokal R, Heimburger O, Krediet R, Plum J, Rodrigues A, Selgas R, Struijk D, Verger C (2005) European best practice guidelines for peritoneal dialysis. 7 Adequacy of peritoneal dialysis. Nephrol Dial Transplant Off Publ Eur Dial Transpl Assoc Eur Ren Assoc 20(Suppl 9):ix24-ix27

14. Vanbelleghem H, Vanholder R, Levin NW, Becker G, Craig JC, Ito S, Lau J, Locatelli F, Zoccali C, Solez K, Hales M, Lameire N, Eknoyan G (2007) The kidney disease: improving global outcomes website: comparison of guidelines as a tool for harmonization. Kidney Int 71:1054-1061

15. La prescrizione dialitica in dialisi peritoneale, Best Practice, Nephro MEET, Società Italiana di Nefrologia. https://www.nephr omeet.com

16. Bannay A, Chaignot C, Blotiere PO, Basson M, Weill A, Ricordeau P, Alla F (2016) The best use of the Charlson comorbidity index with electronic health care database to predict mortality. Med Care 54:188-194

17. Li PK, Szeto CC, Piraino B, de Arteaga J, Fan S, Figueiredo AE, Fish DN, Goffin E, Kim YL, Salzer W, Struijk DG, Teitelbaum I, Johnson DW (2016) ISPD peritonitis recommendations: 2016 update on prevention and treatment. Perit Dial Int J Int Soc Perit Dial 36:481-508

18. Van Biesen W, Williams JD, Covic AC, Fan S, Claes K, Lichodziejewska-Niemierko M, Verger C, Steiger J, Schoder V, Wabel P, Gauly A, Himmele R (2011) Fluid status in peritoneal dialysis patients: the European Body Composition Monitoring (EuroBCM) study cohort. PLoS One 6:e17148

19. van Loon IN, Bots ML, Boereboom FTJ, Grooteman MPC, Blankestijn PJ, van den Dorpel MA, Nube MJ, Ter Wee PM, Verhaar MC, Hamaker ME (2017) Quality of life as indicator of poor outcome in hemodialysis: relation with mortality in different age groups. BMC Nephrol 18:217

20. Korevaar JC, Merkus MP, Jansen MA, Dekker FW, Boeschoten EW, Krediet RT (2002) Validation of the KDQOL-SF: a dialysistargeted health measure. Qual Life Res Int J Qual Life Asp Treat Care Rehabil 11:437-447

21. Mazairac AH, de Wit GA, Grooteman MP, Penne EL, van der Weerd NC, den Hoedt CH, Levesque R, van den Dorpel MA, Nube MJ, ter Wee PM, Bots ML, Blankestijn PJ (2013) Effect of hemodiafiltration on quality of life over time. Clin J Am Soc Nephrol 8:82-89

22. Faruque LI, Wiebe N, Ehteshami-Afshar A, Liu Y, Dianati-Maleki N, Hemmelgarn BR, Manns BJ, Tonelli M (2017) Effect of telemedicine on glycated hemoglobin in diabetes: a systematic review and meta-analysis of randomized trials. CMAJ Can Med Assoc J journal de l'Association medicale canadienne 189:E341-E364

23. Brugts JJ, Manintveld OC, van Mieghem N (2018) Remote monitoring of pulmonary artery pressures with CardioMEMS in patients with chronic heart failure and NYHA class III: first experiences in the Netherlands. Neth Heart J Mon J Neth Soc Cardiol Neth Heart Found 26:55-57

24. Eurlings C, Boyne JJ, de Boer RA, Brunner-La Rocca HP (2019) Telemedicine in heart failure-more than nice to have? Neth Heart J Mon J Neth Soc Cardiol Neth Heart Found 27:5-15

25. Milan Manani S, Crepaldi C, Giuliani A, Virzi GM, Garzotto F, Riello C, de Cal M, Rosner MH, Ronco C (2018) Remote monitoring of automated peritoneal dialysis improves personalization of dialytic prescription and patient's independence. Blood Purif 46:111-117

26. Milan Manani S, Rosner MH, Virzi GM, Giuliani A, Berti S, Crepaldi C, Ronco C (2019) Longitudinal experience with remote monitoring for automated peritoneal dialysis patients. Nephron 142:1-9

27. Walton MK, Powers JH 3rd, Hobart J, Patrick D, Marquis P, Vamvakas S, Isaac M, Molsen E, Cano S, Burke LB (2015) Clinical outcome assessments: conceptual foundation-report of the ISPOR clinical outcomes assessment-emerging good practices for outcomes research task force. Value Health J Int Soc Pharmacoecon Outcomes Res 18:741-752

28. Minatodani DE, Chao PJ, Berman SJ (2013) Home telehealth: facilitators, barriers, and impact of nurse support among high-risk dialysis patients. Telemed J e-Health Off J Am Telemed Assoc 19:573-578

29. Clark RA (2018) Telehealth in the elderly with chronic heart failure: what is the evidence? Stud Health Technol Inform 246:18-23

30. Milan Manani S, Virzi GM, Giuliani A, Crepaldi C, Ronco C (2019) Catheter-related infections in peritoneal dialysis: comparison of a single center results and the literature data. J Nephrol 32:837-841

31. Nayak KS, Ronco C, Karopadi AN, Rosner MH (2016) Telemedicine and remote monitoring: supporting the patient on peritoneal dialysis. Perit Dial Int J Int Soc Per Dial 36:362-366

32. Gallar P, Vigil A, Rodriguez I, Ortega O, Gutierrez M, Hurtado J, Oliet A, Ortiz M, Mon C, Herrero JC, Lentisco C (2007) Two-year experience with telemedicine in the follow-up of patients in home peritoneal dialysis. J Telemed Telecare 13:288-292

33. Sanabria M, Buitrago G, Lindholm B, Vesga J, Nilsson LG, Yang D, Bunch A, Rivera A (2019) Remote patient monitoring program in automated peritoneal dialysis: impact on hospitalizations. Perit Dial Int J Int Soc Perit Dial 39:472-478

34. He T, Liu X, Li Y, Wu Q, Liu M, Yuan H (2017) Remote home management for chronic kidney disease: a systematic review. J Telemed Telecare 23:3-13

35. Martinez Garcia MA, Fernandez Rosales MS, Lopez Dominguez E, Hernandez Velazquez Y, Dominguez Isidro S (2018) Telemonitoring system for patients with chronic kidney disease undergoing peritoneal dialysis: usability assessment based on a case study. PLoS One 13:e0206600

Publisher's Note Springer Nature remains neutral with regard to jurisdictional claims in published maps and institutional affiliations. 